\title{
Moiré Angle Dependent Excitonic Absorption in Twisted Bilayer WSe2 by EELS
}

Steffi Woo ${ }^{1}$, Robert Schneider ${ }^{2}$, Johann Preuß ${ }^{2}$, Ashish Arora ${ }^{2}$, Benjamin Carey ${ }^{2}$, Steffen Michaelis ${ }^{2}$, Alberto Zobelli ${ }^{3}$, Rudolf Bratschitsch ${ }^{2}$ and Luiz H. G. Tizei ${ }^{4}$

${ }^{1}$ Laboratoire de Physique des Solides/CNRS, Université Paris-Saclay, United States, ${ }^{2}$ Institute of Physics and Center for Nanotechnology, University of Münster, Germany, ${ }^{3}$ Laboratoire de Physique des Solides/CNRS, Université Paris-Saclay, France, ${ }^{4}$ Université Paris-Saclay, CNRS, Laboratoire de Physique des Solides, 91405, Orsay, France, France

Atomically-thin layers of semiconducting transition metal dichalcogenides (TMDCs), such as tungsten diselenide ( $\mathrm{WSe}_{2}$ ), have attracted considerable interest because of new properties that can be obtained when artificially fabricated into van der Waals homo- or heterostructures. Recent interest in magic-angle twisted bilayer graphene for the appearance of flat bands [1] has naturally propelled moiré superlattices of TMDCs as a viable candidate for seeking similar phenomena [2]. Due to interlayer coupling, bulk and multi-layered TMDCs are indirect gap semiconductors, while their monolayers exhibit a crossover to a direct band gap. It has been demonstrated that the interlayer coupling strength in homostructures is also sensitive to the twist angle in the cases of bilayer $\mathrm{MoS}_{2}$ [3] and $\mathrm{WS}_{2}$ [4]. In this work, the combined high spatial and spectral resolution of aberration-corrected scanning transmission electron microscopy (STEM) and monochromated electron energy-loss spectroscopy (EELS) in the low-loss regime in the energy range of $0-6 \mathrm{eV}$ are used to investigate the excitonic response of atomically-thin WSe2, specifically in twisted bilayer $\mathrm{WSe}_{2}$ as a function of moiré angle.

Few-layered $\mathrm{WSe}_{2}$ flakes were mechanically exfoliated from a synthetic bulk crystal and transferred onto a $\mathrm{Si}_{3} \mathrm{~N}_{4} \mathrm{TEM}$ grid with periodic micron-sized holes. Atomically-resolved imaging has been performed on a Nion UltraSTEM200 operated at $60 \mathrm{keV}$ and monochromated EELS performed on a modified Nion HERMES-S200 (also known as ChromaTEM) operated at $60 \mathrm{keV}$ with the sample cooled using liquid nitrogen $(\mathrm{T} \approx 150 \mathrm{~K})$. In addition to freestanding WSe2 monolayers, fragments of bilayers and trilayers with variable twist angle between $0-30^{\circ}$ are also routinely observed [Fig. 1(a-c)] due to folding during the mechanical exfoliation and transfer process. Well-defined hexagonal moiré patterns with nanometer periodicity are evident in the high-angle annular dark-field (HAADF) images for the low twist angles in Fig. 1(b). Differentiating the stacking order of the bilayers between $A^{\prime} A^{\prime}(2 H)$ and AA (R-type) stacking was done by comparison with multislice image simulations, assessment of the fold orientation in the case of folded bilayers, and low-angle annular dark-field imaging. With the exception of the $0^{\circ}$ twist angle (i.e. AA $\times$ stacking [Fig. 1(a,d)]), the majority of the twisted bilayers observed exhibit AA (R-type) stacking.

The excitonic absorption signatures of these nanometric twisted bilayers from low-loss EELS are compared to the WSe2 monolayer and trilayer counterparts of zero twist angle in Fig. 1(e). The spectra demonstrate a good general correspondence to the thickness dependence of the $\mathrm{A}, \mathrm{B}$, and $\mathrm{C}$ exciton resonances, namely a pronounced decrease in $\mathrm{C}$ exciton energy with number of layers [5]. Focusing only on the bilayers, the relative energy difference between $\mathrm{A}$ and $\mathrm{B}$ excitons, a measure corresponding to the valence band splitting, is higher for the zero-twist. This reflects an additional contribution to the split bands, other than the spin-orbit splitting, of interlayer coupling energy in the case of AA' stacking [6], 
further corroborating AA-stacking for majority of the twisted bilayers. Comparing different twist angles in the bilayers also show sizable blueshifts in the $\mathrm{C}$ exciton energy up to $200 \mathrm{meV}$, which subsequently alter drastically the spectral shape between the B-C excitonic transitions, with extremes between the zerotwist and towards the anti-aligned $\left(28^{\circ}\right)$ case suggesting underlying differences in interlayer coupling with respect to the moiré angle [7].
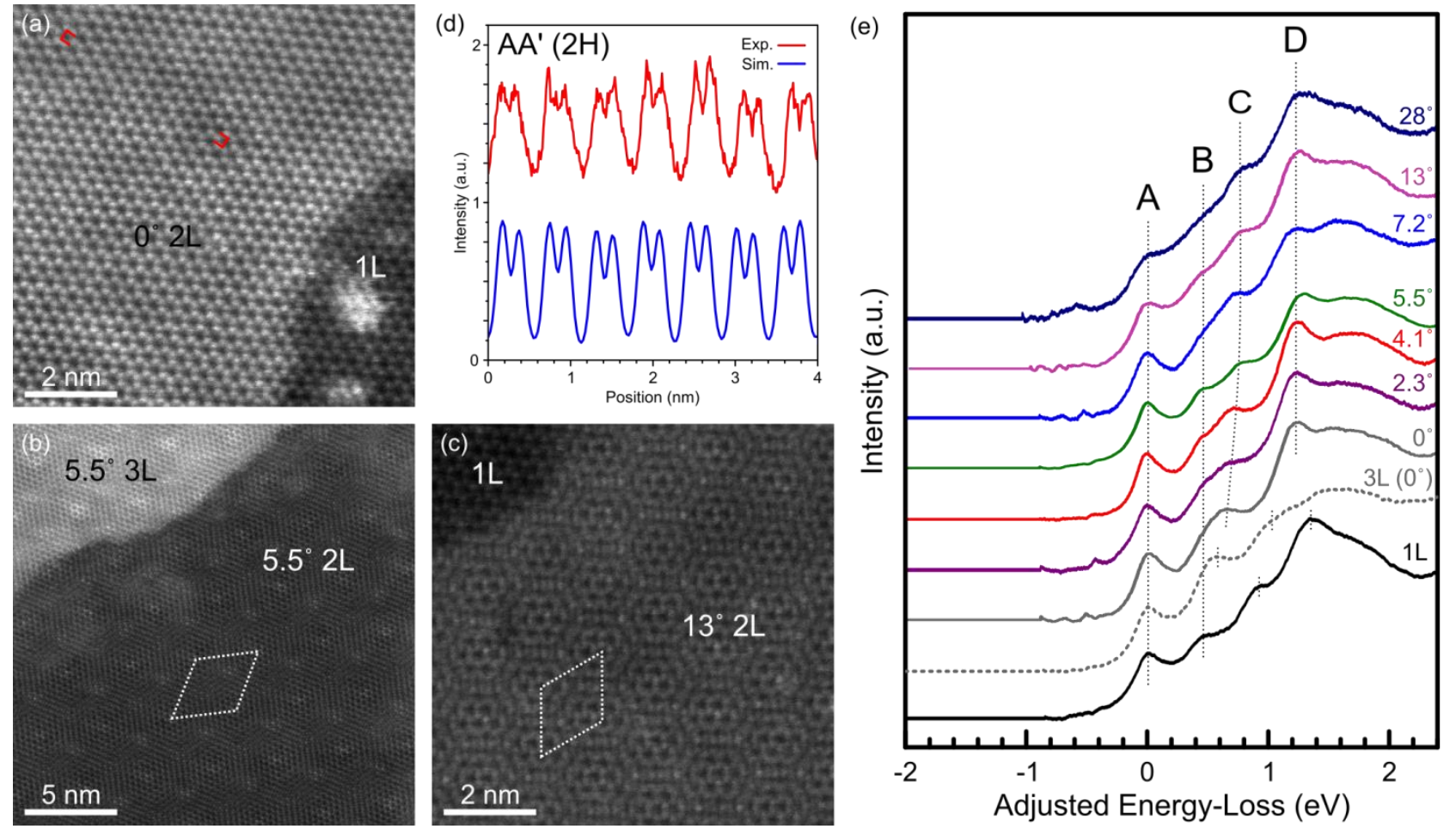

Figure 1. STEM-HAADF images of WSe2 bilayers with (a) $0^{\circ}$, (b) $5.5^{\circ}$, and (c) $13^{\circ}$ twist angle, measured from their image fast-Fourier transform, with the dotted lines marking the moiré unit cell in (b,c). (d) Intensity line profiles comparing experimental and simulated images along the selected area marked by red square brackets in (a). (e) Monochromated EELS spectra from twisted bilayers (2Ls) with various moiré angles compared to a representative monolayer (1L) and trilayer (3L) with zero twist. Spectra are offset in energy relative to the A exciton, and the dotted lines are to help guide the eye in the different exciton energy positions.

\section{References}

[1] Cao, Y., Fatemi, V., Fang, S. et al. Nature 556, 43-50 (2018).

[2] Jin, C., Regan, E.C., Yan, A. et al. Nature 567, 76-80 (2019).

[3] Castellanos-Gomez, A., van der Zant, H.S.J. and Steele, G.A. Nano Res. 7, 572-578 (2014).

[4] Yan, W., Meng, L., Meng, Z., Weng, Y., Kang, L. and Li, X. The Journal of Physical Chemistry C 123 (50), 30684-30688 (2019).

[5] Arora, A., Koperski, M., Nogajewski, K., Marcus, J., Faugeras, C. and Potemski, M. Nanoscale 7, 10421 (2015).

[6] Paradisanos, I., Shree, S., George, A. et al. Nat. Commun. 1, 2391 (2020).

[7] The authors acknowledge funding from the ANR, program of future investment TEMPOSCHROMATEM (No. ANR-10-EQPX- 50). This work was supported by the European Union in the Horizon 2020 Framework Program (H2020-EU) under Grant Agreement No. 823717 (ESTEEM3). 